\title{
Agglutination of platelets by a serum factor in the presence of EDTA
}

\author{
E. GOWLAND, H. E. M. KAY, JUDY C. SPILLMAN, AND J. R. WILLIAMSON \\ From the Department of Clinical Pathology, Royal Marsden Hospital, and Chester Beatty Research Institute, \\ Institute of Cancer Research, London
}

SYNOPSIS The platelets of a patient with reticulum-cell sarcoma were found to exhibit agglutinationf and cytopathic changes when her blood was treated with EDTA but not with other anticoagulants Investigation showed that the phenomenon was caused by a serum factor that was probably an antibody.

The occasional development of autoantibodies in patients with neoplastic disease of the reticuloendothelial system is well documented (Dacie, 1962). In this case report we describe the discovery of an antiplatelet antibody in a patient with reticulum-cell sarcoma. The case is remarkable in that a reaction between platelets and antibody was observed only when the anticoagulant employed was EDTA (ethylenediamine tetra-acetate).

\section{CASE REPORT}

A 70-year-old woman was admitted with a history of lumps in the right groin for 18 months. Biopsy of an enlarged inguinal lymph node revealed a reticulum-cell sarcoma of pleomorphic-cell type. She had once received a blood transfusion 15 years previously. In a routine count from a venous sample with EDTA the platelets were found to number $45,000 / \mathrm{cmm}$. These were estimated using the Coulter model A counter by the method described by Williamson (1967). Examination of the blood film, however, showed many clumps of pale-staining platelets, mostly towards the tail of the film, and a repeated blood count using a capillary sample without EDTA gave a platelet concentration of $249,000 / \mathrm{cmm}$, which seemed to be in accordance with the numbers in the blood film.

\section{INVESTIGATIONS AND RESULTS}

The phenomenon was further investigated using a variety of anticoagulants: heparin (Pularin) ${ }^{1} 12.5$ units per $\mathrm{ml}$ of blood; $3 \cdot 8 \%$ wv sodium citrate; disodium and dipotassium EDTA, $2 \% \mathrm{wV}, 1$ volume to 9 volumes of blood. Commercially prepared tubes

${ }^{1}$ Evans Medical Ltd.

Received for publication 27 November 1968. containing EDTA ${ }^{2}$ were also used. Polystyrene con tainers were employed throughout.

The effect of EDTA on the patient's platelets is illustrated in Figure 1. In this experiment sequentiab platelet counts were performed on each of threg specimens of blood treated with EDTA, citrate, of heparin and also on a specimen to which no anti coagulant had been added. Specimens from threê normal individuals, of which one is illustrated $\frac{0}{\circ}$ served as controls. In specimens without an anti coagulant the counts fell gradually for six to eigh? minutes and then decreased rapidly as clotting occurred. In the citrate and heparin samples there was a progressive fall in the apparent platelet con centration probably due to clumping, but this decrease was not of a significantly greater magnitudes than that of normal controls. In normal circum 3 . stances only EDTA effectively prevents this prof gressive clumping as is illustrated by the results in the control blood sample. However, when the patient's blood was treated with EDTA, in the form of either the sodium or the potassium salt, the plate응. let count was severely and rapidly reduced.

In slides of the patient's blood with EDTA ${ }^{\circ}$ stained by May-Grunwald-Giemsa the clumps of platelets were seen to be palely basophilic, and azurophilic granules were very scarce, whereas in the absence of EDTA the platelets were morphoo logically normal.

These changes were observed in greater detail by electron microscopy. Specimens of the patient's0 blood were treated with EDTA and citrate ancP allowed to stand at room temperature for about $9 \mathrm{C}^{\circ}$ minutes. The platelet-rich plasma was then harvested from each, and, after centrifugation, the platelet ${ }^{2}$ Stayne Laboratories Ltd. 


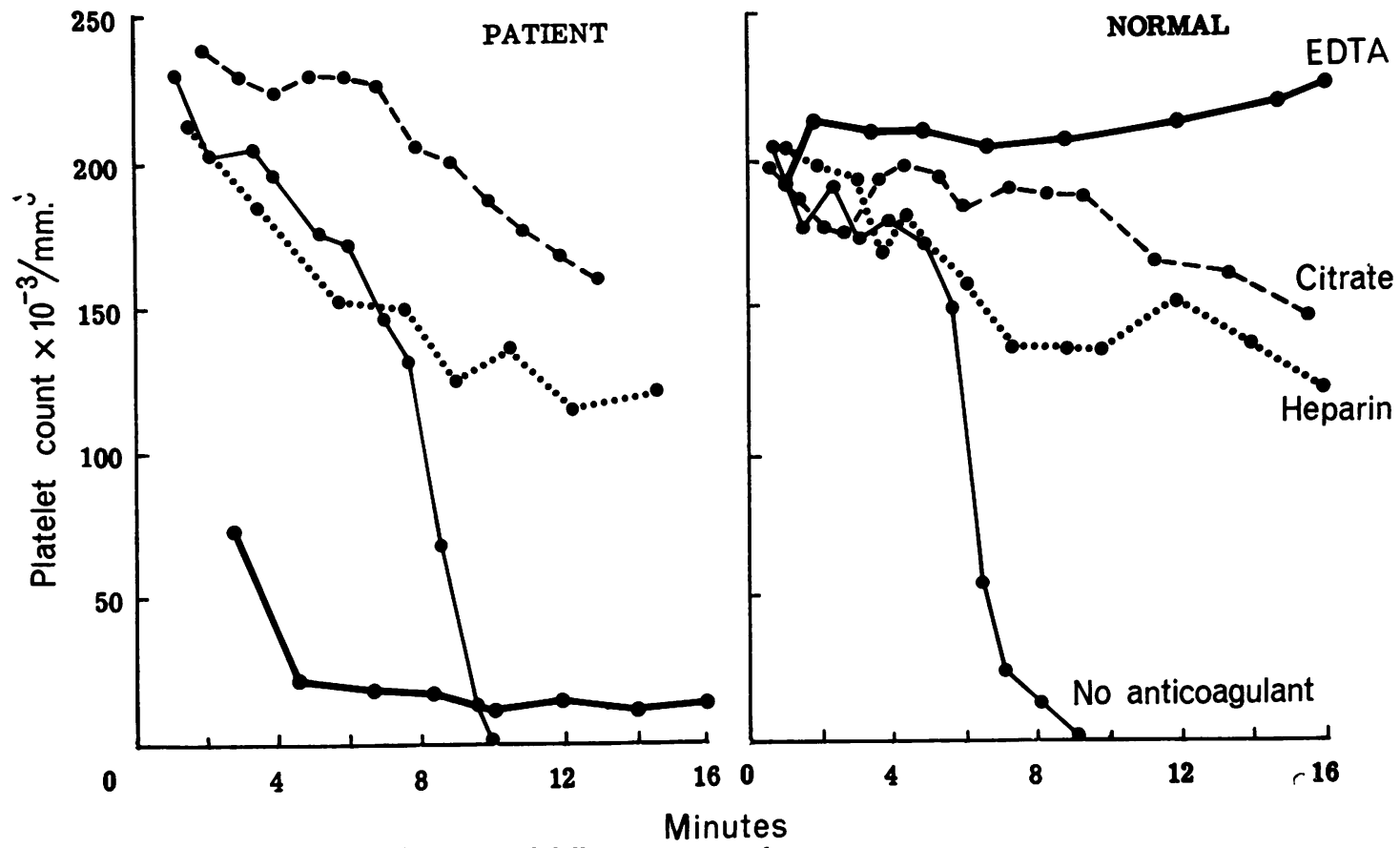

FIG. 1. Effect on apparent platelet count of different anticoagulants.

pellets were fixed in glutaraldehyde. The electron micrographs prepared from these specimens, together with those made from similar preparations of platelets from a normal subject, are shown in Figures 2 to 5 . These show no abnormality of the patient's platelets when incubated in citrated plasma (Fig. 2) but there are fairly profound differences from the normal in EDTA preparations. The control platelets remain discrete, irregularly rounded, and show little loss of the dense $\alpha$-granules which characterize platelets (David-Ferreira, 1964). A few platelets contain small or medium-sized vacuoles (Fig. 3).

The patient's platelets, by contrast (Figs. 4 and 5), are swollen, with consequent disorganization of structure; some platelets contain many small vacuoles and in almost all there is a loss of dense granules that is particularly conspicuous when compared with the platelets in citrate. Platelet aggregation in EDTA has taken the form of focal clustering as distinct from the fairly uniform 'crazy paving' effect in citrate.

Clearly the abnormal reactivity of the patient's platelets could be either intrinsic to the platelets themselves or result from the action of a factor in the plasma. The absence of bleeding manifestations and the ability of the platelets to cause normal clot retraction could be interpreted either way, and the answer was provided very simply by testing the patient's serum with normal platelets.
Serum from the patient was added to an equal volume of blood from each of five individuals of the same group (group 0). Both blood and serum were treated with EDTA and then placed on a roller mixer for 30 minutes, after which a platelet count was determined. The results, which are presented in Table I, show platelet counts that were much lower than would have been expected had no reaction occurred, whereas in control tests with serum form normal subjects the counts obtained were close to expected values. Tests performed with the patient's serum diluted with saline to $1 / 8$ showed a reduction in platelet count of more than $50 \%$. From these results it appeared that the patient's serum contained a factor that caused clumping of normal platelets in the presence of EDTA. This conclusion was confirmed by the results of an experiment in which the test outlined above was repeated using platelet-rich plasma from normal subjects instead of whole blood. Microscopic examination of the specimens after 30 minutes on the roller mixer revealed marked clumping of the platelets that had been treated with the patient's serum, but not of those treated with control serum.

On the premise that the 'antiplatelet' factor present in the patient's serum might be an antibody, we tested protein fractions of her serum for activity. Two fractions were tested: the first comprised the globulins precipitated at $33 \%$ saturation with 


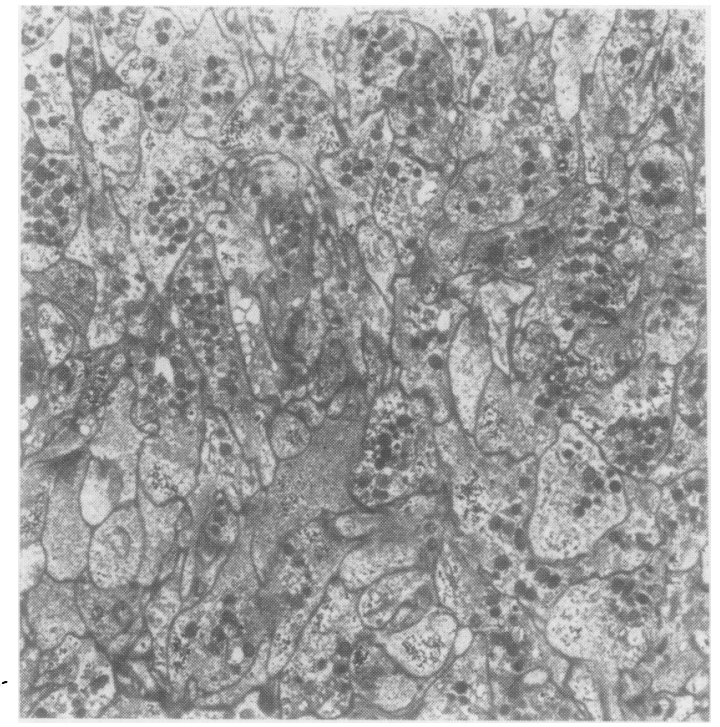

FIG. 2.

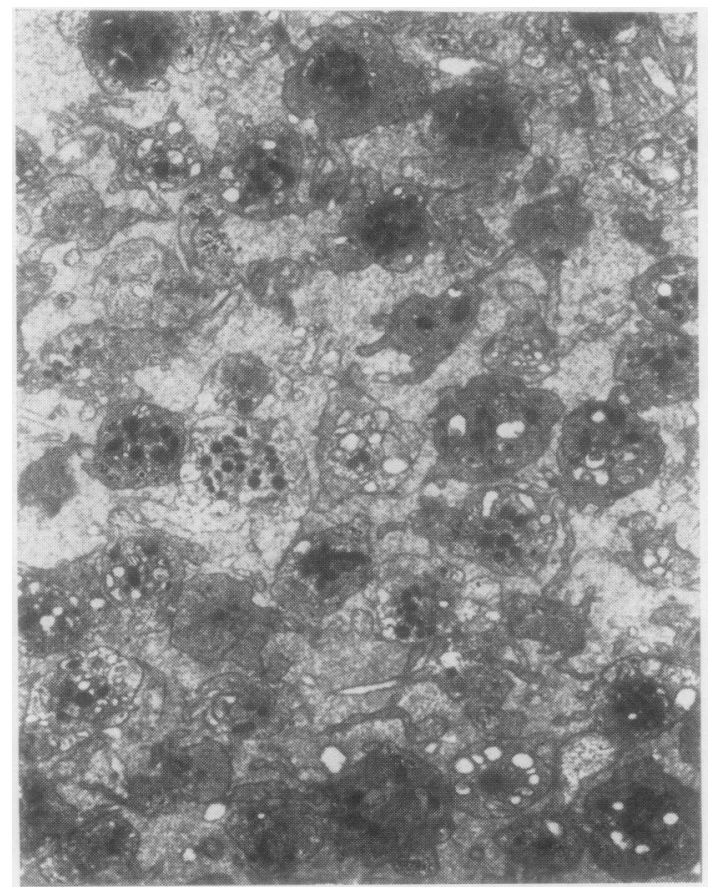

FIG. 3.
FIG. 4 .

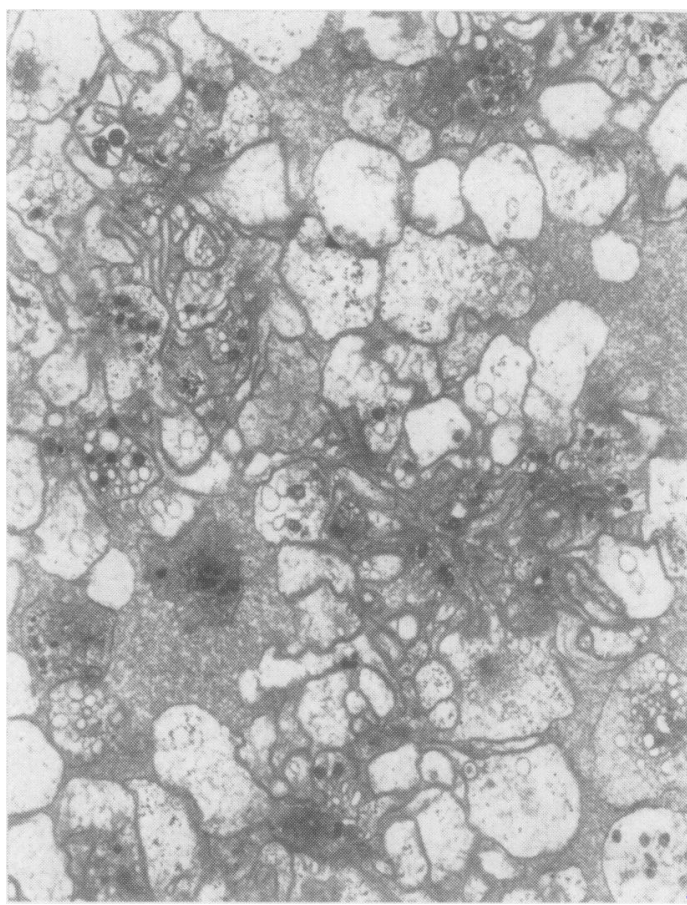

FIG. 2. Patient's platelets in citrated plasma $\times 5,000$.

FIG. 3. Normal platelets in EDTA plasma $\times 5,000$

FIG. 4. Patient's platelets in EDTA plasma $\times 5,000$. 


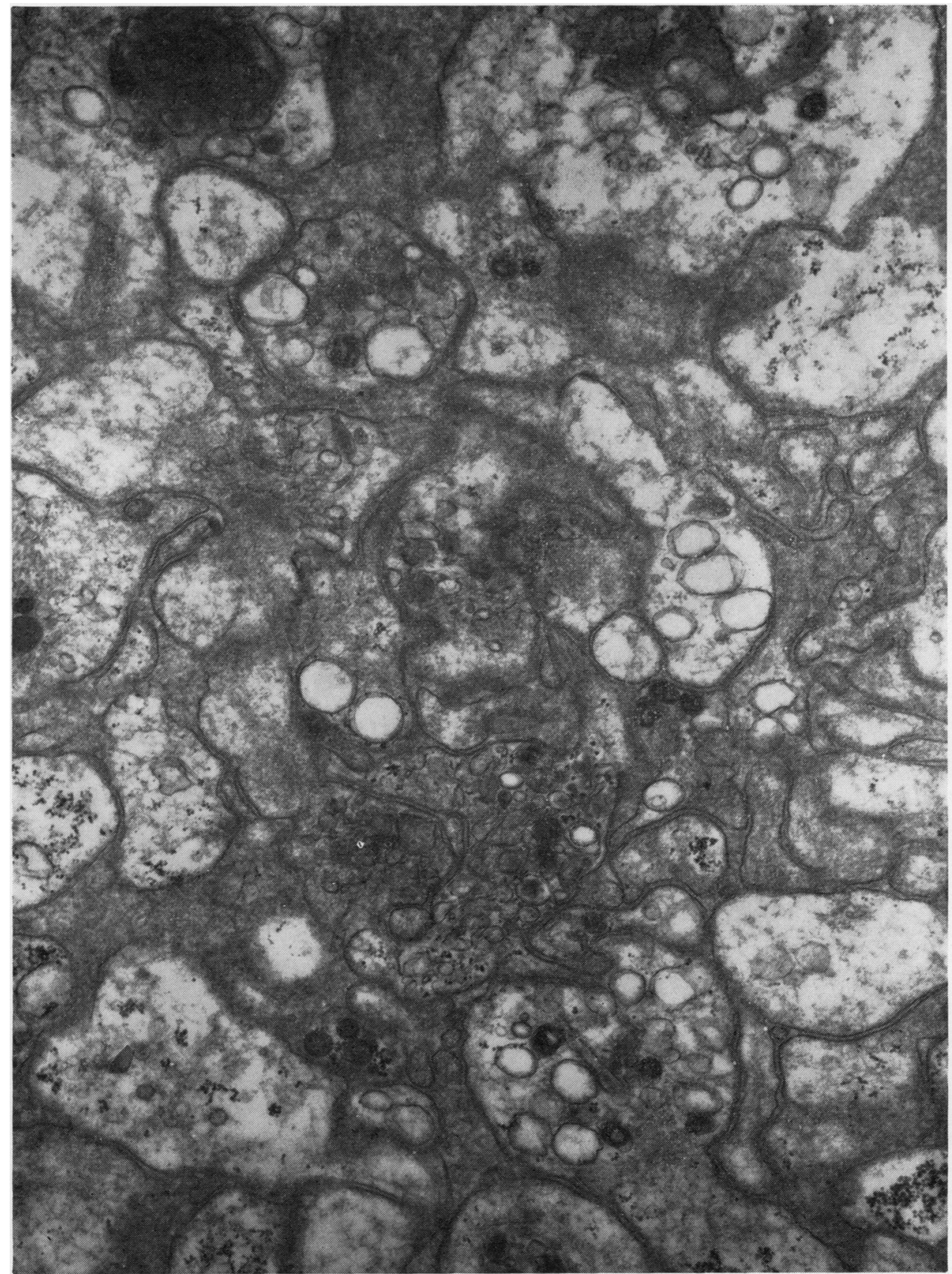

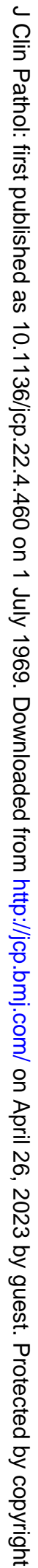

ammonium sulphate ('euglobulin' fraction) and the second those globulins precipitated when the concentration of ammonium sulphate was increased from $33 \%$ to $50 \%$ saturation ('pseudoglobulin' fraction). The 'euglobulin' fraction showed marked activity when treated for its capacity to lower the platelet count of a specimen of normal blood, whereas the 'pseudoglobulin' fraction was inactive (Table II).

These findings suggest that the factor involved was an antibody. Supportive evidence was provided by Dr K. L. Goldsmith at the MRC Blood Group 
TABLE I

\begin{tabular}{|c|c|c|c|c|}
\hline \multirow[b]{2}{*}{$\begin{array}{l}\text { Blood } \\
\text { Donor }\end{array}$} & \multicolumn{4}{|c|}{ EFFECT OF NORMAL AND PATIENT'S SERUM ON PLATELETS FROM FIVE NORMAL SUBJECTS } \\
\hline & $\begin{array}{l}\text { Initial Platelet } \\
\text { Count }\end{array}$ & Serum Added & $\begin{array}{l}\text { Platelet Count after } \\
\text { Addition of Equal Volume } \\
\text { of Serum }\end{array}$ & Percentage Change \\
\hline 1 & 160,000 & Normal & 82,000 & $\begin{array}{r}3 \\
+\quad 3\end{array}$ \\
\hline 1 & 160,000 & Patient's & 19,000 & -76 \\
\hline 2 & 132,000 & Normal & 69,000 & $\begin{array}{r}-4 \\
+\quad 4\end{array}$ \\
\hline 2 & 132,000 & Patient's & 14,000 & -79 \\
\hline 3 & 134,000 & Normal & 72,000 & $\begin{array}{r}7 \\
+\quad 7\end{array}$ \\
\hline 3 & 134,000 & Patient's & 13,000 & -81 \\
\hline 4 & 149,000 & Normal & 74,000 & -1 \\
\hline 4 & 149,000 & Patient's & 23,000 & -69 \\
\hline 5 & 185,000 & Normal & 77,000 & -17 \\
\hline 5 & 185,000 & Patient's & 17,000 & -82 \\
\hline
\end{tabular}

TABLE II

EFFECTS OF PATIENT'S DILUTED SERUM OR SERUM FRACTIONS ON NORMAL PLATELETS

Initial Platelet

Count of Blood

\begin{abstract}
Added Serum
or Fraction
\end{abstract}

Platelet Count after

Adding Equal Volume

of Serum or Fraction

Normal serum
Patient's serum
Patient's serum diluted $1: 2$
Patient's serum diluted $1: 4$
Patient's serum diluted $1: 8$
Patient's serum diluted $1: 16$
Normal 'euglobulin'
Patient's euglobulin
Patient's euglobulin
Patient's 'pseudoglobulin'

77,000

17,000

13,000

22,000

38,000

66,000

94,000

16,000

18,000

82,000
Reference Laboratory where an antiplatelet antibody in the patient's serum was demonstrated by a complement-fixation technique. It does not, of course, follow from this observation that complement fixation is involved in the phenomenon described above; although the morphological changes suggest a complement-mediated lysis it is, in fact, improbable that complement is involved in view of the anticomplementary activity of EDTA.

Three hypotheses may be forwarded to explain the fact that a reaction between antibody and platelets was observed only in the presence of EDTA. The first is that EDTA acts directly on the antibody to bring about its activation. The second proposes that EDTA destroys an inhibitor of the antibody. Neither of these seems likely in the light of present knowledge. The third hypothesis holds that the surface structure, and hence the antigenic determinants of the platelets, might be modified by the anticoagulant. Ethylenediamine tetra-acetate is well known to affect the structure of platelets, causing them to become more spherical and to remain discrete (Zucker and Borrelli, 1954). Furthermore the survival of EDTA-treated platelets after transfusion is poor compared with citrate-treated platelets (Aster, 1966) and some direct or indirect toxic effect upon normal platelets can be presumed. In this instance they were apparently rendered susceptible to the action of an autoantibody of wide but undetermined specificity.

The phenomenon is of interest as a manifestation? of autoimmunity in neoplasia of the reticulo? endothelial system but is not otherwise of patho logical importance. In practice EDTA remains the most satisfactory anticoagulant for platelet estimations, but the chance of anomalous agglutination as in this case, makes examination of the blood films a wise precaution.

\section{ADDENDUM}

More recently a second example of this phenomenoñ was identified in an 82-year-old man with a peptic ulcer previously transfused once five years before $\mathcal{N}$ As before, the agglutination of the platelets, though less pronounced, occurred only in the presence of EDTA. The phenomenon could not in this instanceo be shown with plasma fractions.

\section{REFERENCES}

Aster, R. H. (1966). Transfusion (Philad.), 6, 32.

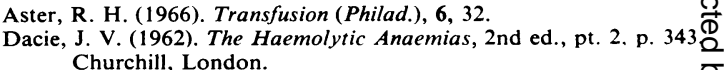

David-Ferreira, J. F. (1964). Int. Rev. Cytol., 17, 99.

Williamson, J. R. (1967). Progr. med. Technique, 4, 118.

Zucker, M. B., and Borrelli, J. (1954). Blood, 9, 602. 